\title{
Maintaining a Sense of Control in Autonomous Vehicles via Auditory Feedback
}

\author{
David Beattie ${ }^{1}$, Lynne Baillie ${ }^{1}$, Martin Halvey ${ }^{1}$, Rod McCall ${ }^{2}$ \\ ${ }^{1}$ Interactive and Trustworthy Technologies Research Group, Glasgow Caledonian University, Glasgow, \\ UK \\ ${ }^{2}$ University of Luxembourg, Interdisciplinary Centre for Security, Reliability and Trust, Luxembourg \\ david.beattiedgcu.ac.uk, l.baillie@gcu.ac.uk, martin.halvey@gcu.ac.uk, \\ roderick.mccalleuni.lu
}

\begin{abstract}
This paper presents the findings from an observational field study conducted with 8 car drivers. The study attempted to create a taxonomy of sounds that present information to people whilst driving. We also aimed to determine whether participants noticed these sounds as they occurred and whether they paid attention to them. Furthermore, we asked the participants subjective questions regarding particular sonic attributes and their ability to catch driver's attention. It was concluded that although certain sounds occur regularly, differing levels of attention are given to each depending on the information they present. Our study also revealed that while all sonic attributes play an impact in catching driver's attention, some aspects are more noticeable than others. We conclude with a discussion of our future directions with regards to the findings obtained from our observational field study and outline the plan for our next study.
\end{abstract}

Index Terms: Auditory feedback, autonomous vehicles, subjective observational field study.

\section{Introduction}

Since the invention of the internal combustion engine there have been a number of technological advances that have propelled the development of the automobile [1]. In the early stages of vehicle development it was breakthroughs in manufacturing that made notable marks on the automotive industry allowing massproduction of automobiles such as the Model T Ford [2]. Innovation still continues at a rapid rate improving vehicles for the drivers' benefit [3]. Modern car development is constantly evolving and this evolution stems from a variety of different motivations such as the need to increase safety [4], improve situational awareness (SA) [10] and present non-primary driving information [5]. Advancements such as collision warning and avoidance systems; Adaptive Cruise Control (ACC); lane departure warning systems and automated parking all lead towards a more automated driving experience where the driver is no longer in full control of their vehicle $[6,7,8]$. It is important for drivers to be made aware of what these automated systems are doing and this information must be presented in an appropriate manner to ensure the primary driving tasks are not hindered, drivers are not distracted and to ensure a sense of control is maintained [9, 10]. As systems such as ACC and others become commonplace and ultimately vehicles become fully autonomised $[11,12]$, it is important to address what effect such a loss of control has on user perception.
This work investigates how advancements in vehicular technology must take into account user perception in order to ensure the sense of vehicular control is not lost. The goals of this research are: (1) to investigate how a loss of control in autonomous vehicles will affect drivers, (2) to develop auditory feedback that can be provided to drivers to effectively augment this loss of control encountered and (3) determine how this auditory feedback can alleviate any anxiety introduced when travelling in autonomous vehicles. This paper presents an outline of the issues regarding current vehicular technological advancements and its impact on a drivers perceived level of control and discusses possible solutions to this perception by augmenting the driving experience with auditory feedback.

\section{Context and Motivation}

Car development has progressed substantially with the inclusion of computerised systems. They have not only improved mechanical efficiency with the development of engine management systems, electronic ignition and fuel-injection systems but occupant safety has been increased via the addition of anti-lock braking systems (ABS), improved traction control and power assisted steering (PAS) [18]. It is also interesting to point out that the inclusion of computerised systems not only improves road safety and vehicle efficiency, but they can also create a more enjoyable driving experience [13].

Stanton and Marsden [14] note that there is an "unstoppable force" in current vehicle engineering to computerise and automate functions related to driving and suggest that the driving task is changing. Because of this, there is particular interest in using computing to accomplish primary driving tasks in the development of fully autonomous vehicles. For example the DARPA Urban Challenge [15] sees several notable proponents from various research groups take part in an attempt to successfully develop autonomous vehicles, for the purpose of negotiating urban environments with both manned and unmanned vehicles. Winning the 2005 challenge led Stanford University to develop a driverless car in collaboration with Google [16].

These competitions present a platform for the development of fully automated vehicles. However, there is a distinct lack of consideration regarding the control shift computerised systems introduce and the impact this may have on the driver [17]. While the inclusion of computerised systems can create a more enjoyable driving experience [14], as control is shifted away from the driver and vehicles become autonomous, there is a limit to the enjoyment felt. This is because the travelling experience for the driver and passengers is not taken into account [18]. 
Kraus et al. [18] note two ways in which drivers and passengers are not being taken into account: 1. Due to their planned trajectories, the driving style of autonomous vehicles feels particularly unnatural in comparison to human driving styles. 2 . There is a distinct lack of feedback for passengers making them unable to determine whether an autonomous vehicle is aware of particular traffic situations and whether or not evasive action should be performed. Therefore, by not providing the necessary feedback to both the driver and the passengers in autonomous vehicles then a perceived distinct loss of control could be experienced. This loss must be quantified to determine its importance for providing a more natural driving experience in autonomous vehicles.

In-vehicle technologies in current vehicles catering for secondary driving tasks such as GPS navigation, in-car entertainment systems and other non-primary information often do not present their feedback in the most appropriate manner [4]. This can significantly impede on the primary driving task [14]. In these circumstances auditory feedback can be used effectively to reduce any distraction incurred when operating these secondary functions [5]. Automation of the primary driving tasks; steering, gear-changing, acceleration and braking has the ability to reduce the mental workload for drivers to a point where they simply monitor the systems operating the vehicle [8]. One benefit of this is the ability to represent information in vehicles visually without any impact on driving performance. However, auditory feedback will also be required to provide situational awareness of what is happening both inside and outside the autonomous vehicle in order to augment the primary driving task so users feel in control.

\section{Methodology}

The purpose of a fully autonomous vehicle is to remove the need for the driver to perform primary driving tasks. Doing so however means control is no longer in the drivers' hands. One way to alleviate this loss of control is to substitute the primary driving tasks with auditory feedback. In order to determine how this supplementary auditory feedback will reintroduce a sense of control it is first important to understand the extent to which auditory feedback in current vehicles helps drivers to feel in control. More specifically, which particular sounds, notifications and functions achieve this feeling of control. A preliminary field study was conducted to investigate sounds in current vehicles and user opinions of these sounds in relation to information conveyance.

\subsection{Preliminary Field Study}

A small-scale field study was conducted to collect all of the sounds present in current vehicles that present information to the driver. Our main goal in this study was to acquire data that would help categorise vehicle sounds in terms of how much importance was given to them. Furthermore, it sought to establish which particular sonic attributes were perceived to be more/less important and would provide insight into user preferences regarding current vehicle sounds and any possible additions.

The study took place in and around the cities of Edinburgh and Glasgow. Our participants were recruited via word of mouth, agreeing informally to undertake the study. They were then emailed with an outline of the study and given the opportunity to opt in or out. We gathered 8 participants who had an age range of 26 - 35, with 2 being female and 6 being male. They all had varying degrees of driving experience having held their licenses for a range of $5-10$ years. 4 of the users owned their own vehicle while the rest had regular access to a family/work car. Average weekly drive time amongst participants was between 3 -6 hours with the most common journeys being work related, such as commuting, followed by journeys to recreational activities. There were 5 common car types ranging from $4-9$ years old with a value of $£ 3,000-£ 10,000$.

\subsection{Procedure}

Each study was conducted with a car participants owned or had regular access to. They were asked to travel a regularly driven route for a minimum of 15 minutes. As the purpose of the field study was to capture the sounds that help to convey information to drivers, it was imperative that the driving task not be impeded by any invasive procedures. A marking system was deduced and used in conjunction with a pre-determined taxonomy of expected sounds. This enabled the notation of sound occurrences to be quick and easy and ensured minimal driver distraction. Each different sound was marked on a checklist using a, "|" symbol as they occurred. If participants noticed a sound then the, "|" symbol was encapsulated in brackets: "( | )". Additionally, it was highlighted that participants must notify the observer of any sounds they identified that were not noticed by the observer. If this occurred then a "+" was used: " $(+)$ ". All sounds were recorded using a portable two channel recording device. Our sound taxonomy was created from sounds collected during 2 pilot tests. After noting individual sounds that occurred during the 15-minute pilot tests we concluded that there were 20 common interior sounds as listed below:

Table 1: Taxonomy of Sounds

\begin{tabular}{|c|c|c|}
\hline $\begin{array}{l}\text { Primary } \\
\text { Functions }\end{array}$ & $\begin{array}{l}\text { Secondary } \\
\text { Functions }\end{array}$ & $\begin{array}{l}\text { Exterior } \\
\text { Sounds }\end{array}$ \\
\hline Horn & Mirror Adjustment & $\begin{array}{c}\text { Continuous } \\
\text { Road Tyre } \\
\text { Noise }\end{array}$ \\
\hline Indicator & $\begin{array}{c}\text { Turning ON/OFF } \\
\text { Lights } \\
\end{array}$ & Traffic Lights \\
\hline Ignition & Car Heater/Fan & $\begin{array}{c}\text { External Car } \\
\text { Horns }\end{array}$ \\
\hline Clutch & Radio/CD Player & $\begin{array}{c}\text { Rumble Strip } \\
\text { Noise }\end{array}$ \\
\hline Braking & $\begin{array}{c}\text { Electric Window } \\
\text { Adjustment }\end{array}$ & $\begin{array}{c}\text { Emergency } \\
\text { Services }\end{array}$ \\
\hline Acceleration & Door Locks & \\
\hline \multirow[t]{2}{*}{ Gear Changing } & $\begin{array}{c}\text { Door } \\
\text { Opening/Closing } \\
\end{array}$ & \\
\hline & Seat Adjustments & \\
\hline
\end{tabular}

Before setting off the participants were briefed about the study and its purpose then asked to fill out a pre-evaluation questionnaire containing demographic questions. During this time the observer positioned the recording device between the front passenger seats and set to record $320 \mathrm{kbps}$ MP3 files. Participants were then asked to enter the car to begin the study. 
Upon completion of the participant's regular driving route they were asked politely to stop in a safe place at which point the audio recording was stopped. Once safely stopped the postevaluation questionnaire was then given to each participant for completion. This evaluation featured both qualitative and quantitative questions and provided us with a rich set of data related to vehicular sound and user opinion.

\section{Initial Findings}

The field study was conducted in order to capture any additional sounds which we had not yet identified on our taxonomy. It would enable us to determine the extent to which drivers noticed these sounds. Additionally, the study would provide an insight into what extra notifications users would like to have presented sonically. Finally it allowed us to establish which particular sonic attributes were most useful at catching participants' attention.

\subsection{Newly Obtained Sounds}

During the study, whilst the participants drove their regular route, the observer marked sound occurrences relating to the predetermined taxonomy. Upon completion of all studies we acquired 14 new sounds. 14 out of the 21 sound occurrences were highlighted by the participants themselves whilst undertaking the study. These sounds were either mechanical notifications e.g. service requirement notifications or related to vehicle operations. Below is a table of sounds collected and their occurrences.

Table 2: Sounds Captured During Field Study

\begin{tabular}{|c|c|c|}
\hline $\begin{array}{c}\text { New Sounds } \\
\text { Occurred }\end{array}$ & $\begin{array}{c}\text { Amount of Times } \\
\text { Occurred }\end{array}$ & $\begin{array}{r}\text { Identified by } \\
\text { Participant }\end{array}$ \\
\hline $\begin{array}{l}\text { Gear Box Noise } \\
\text { (crunch) }\end{array}$ & 1 & 1 \\
\hline $\begin{array}{c}\text { Washer Fluid } \\
\text { Alert }\end{array}$ & 1 & 1 \\
\hline $\begin{array}{c}\text { Service } \\
\text { Notification }\end{array}$ & 2 & 0 \\
\hline $\begin{array}{l}\text { Brake Pad } \\
\text { Replace } \\
\text { Notification }\end{array}$ & 1 & 1 \\
\hline Other Cars & 4 & 3 \\
\hline Hand Brake & 4 & 2 \\
\hline Engine Idle & 2 & 0 \\
\hline Seat Belt Alert & 2 & 1 \\
\hline Speed Bumps & 2 & 2 \\
\hline Stall & 1 & 0 \\
\hline $\begin{array}{l}\text { Reverse Gear } \\
\text { Notification }\end{array}$ & 2 & 1 \\
\hline Parking sensor & 1 & 1 \\
\hline $\begin{array}{c}\text { Tyre pressure } \\
\text { indicator }\end{array}$ & 1 & 0 \\
\hline Passengers & 3 & 0 \\
\hline
\end{tabular}

\subsection{Sound Occurrence Identification/Noticeability}

The results acquired from the observation section of our study produced a number of subjective findings that are summarised in this section. Firstly, there were a total of 258 sound occurrences produced from the 8 studies. Out of these 258 , the total amount of sounds noticed was 133 , the total sounds identified were 24 giving 101 remaining sounds neither noticed nor identified. From this it can be concluded that $60.85 \%$ of all sound occurrences were either noticed or identified. This result highlights the importance of sound as a means of notifying drivers to specific information.

Figure 1 displays 3 sounds from our taxonomy that correspond to interior and exterior sounds with differing results. Firstly, it displays two interior noises, (Horn, Gear Changing) and shows their relative user identification/noticed amounts. Overall the horn sound occurred 8 times. 7 of these times it was observed that the participants noticed the sound and 1 of these times a participant identified the sound personally. In contrast, the gear changing sound occurred 38 times but was noticed 10 times and identified 3 meaning 25 occurrences of the sound were neither noticed nor identified. This points towards a prioritisation of sounds depending on the function to which they are attached. For instance, the horn is an immediate primary function that occurs less often in comparison to the regularity of changing gears. It can be inferred that the regularity of certain functions are noticed less in comparison to more immediate, less frequently occurring sounds.

Secondly, we compared an exterior sound (Road Tyre Noise) with the interior sounds to determine its total occurrence and its identification/notification amounts. Road tyre noise events occurred a total of 24 times throughout the study of which 2 were identified and 5 were noticed. Therefore, 17 occurrences of road tyre noise were inferred as unnoticed. As this sound is continuous, users may not consider it a sound to pay a considerable amount of attention to. Perhaps only would attention be paid if a particular sonic attribute of the road tyre noise changes during a journey. However, as the sound was identified and also noticed, further research is needed in order to determine scenarios in which participants pay greater attention to this particular sound.

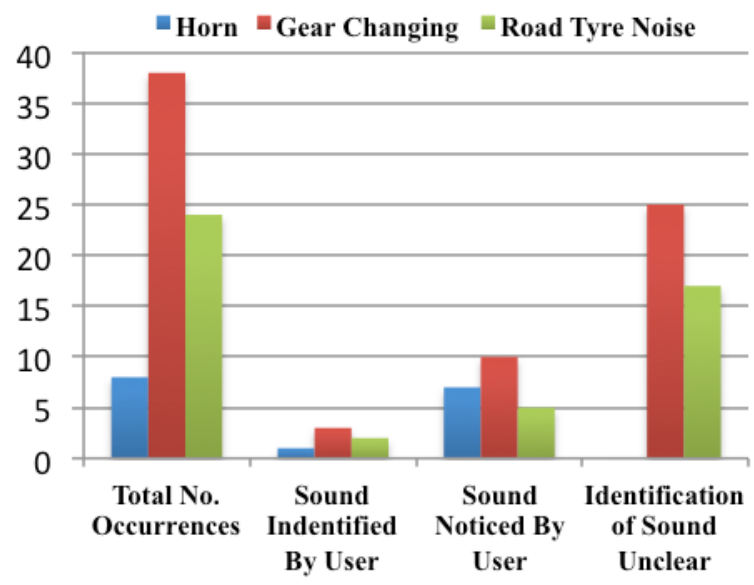

Figure 1: Level Of Sound Occurrences In Comparison to Identification and Noticeability 


\subsection{Sonic Parameter Findings}

After analysing the sound occurrences from the subjective results of the observational study we asked participants to provide us with their opinions regarding the particular sonic attributes that were most useful in catching their attention.

Our findings suggest that the attribute "Volume" was most significant in catching the participants' attention with a value of $30.61 \%$. The "Pitch" and "Duration" attributes were joint second in terms of significance with a value of $21.09 \%$. "Timbre", "Repetition" and "Melody" were least significant. It is surprising that "Repetition" did not score higher in terms of noticeability as the indicator notification sounds exploits this attribute, occurring 40 times during our study and being noticed 30 times. Our next study will attempt to delve further into the effects of sonic parameters in relation to gaining the attention of participants.

It can be concluded from our findings that the attributes volume, pitch and duration of a particular sound are important to exploit in order to ensure a sound is attention grabbing. We intend to investigate this point further by exploring these particular attributes further in a virtual driving simulator.

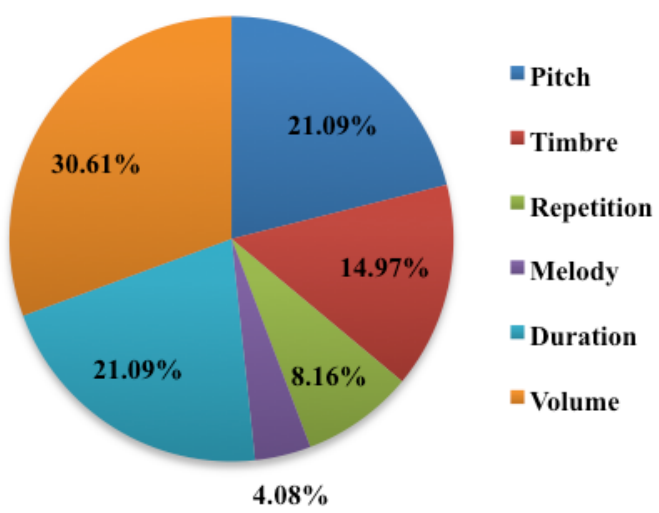

Figure 2: Sonic Attributes Most Useful For Catching Attention

\subsection{Qualitative Results}

Our post evaluation questionnaire provided the chance to obtain some qualitative responses from our participants relating to a number aspects regarding sound. For the purpose of this paper we will briefly discuss opinions regarding the addition and removal of particular sounds not covered during the observational field study.

With regards to the inclusion of sounds, a number of users hinted towards feedback from technology already common place in modern high-end vehicles. Some common responses were sounds from GPS navigation, road information (weather/traffic condition updates) and parking sensors. As these sounds were not included in our field observation it is important to study the sounds that relate to these systems and to ascertain users opinions of them. As well as the inclusion of sounds we asked participants about sounds they wished to have removed from their vehicles. 7 out of 8 users would have liked less exterior noise within their vehicle stating that it interferes with activities such as music listening and conversing with passengers. Some responses from our participants were;

\section{"A quieter car would be more pleasurable to drive. Feels more secure" - User 8 \\ "I like to hear the engine to know how the car 'feels' when I'm driving". - User 4}

This points towards participants attaching importance to mechanical sounds related to primary driving task such as engine revs. Furthermore it suggests that a reduction in road tyre noise would benefit drivers when they wish to engage in activities unrelated to driving.

\section{Conclusion}

Our observational field study is now complete and we have begun to analyse the findings. So far, we have received some insightful results that highlight a number of interesting possibilities for auditory feedback in autonomous vehicles. We have yet to fully analyse all of the collected data and will continue with the categorisation of the interior and exterior sounds captured.

So far it can be concluded that sound contributes significantly when used to present information within vehicles. This study has enabled us to capture a wider range of sounds that can be added to our pre-existing taxonomy of sounds. Furthermore it has helped us to categorise sounds that occur in vehicles relating to the importance put on them by drivers. The sounds can also be further categorized by their sound source relative to the vehicle (interior/exterior). Restrictions on sound design for autonomous vehicles will be vastly different to current vehicles due to differing safety implications. A taxonomy of sounds and how users respond to these currently will serve as a thorough basis from which sounds can be created for autonomous vehicles in future situations. Furthermore, the effect of individual sonic attributes on drivers will change, which is an important consideration when designing sounds that do not need to adhere to such strict safety restrictions.

\section{Future Work}

The results from the observational field study have provided some interesting considerations with regards to the types of sounds currently noticed by drivers. It is important to use these results to determine whether current vehicular sounds are effective within an autonomous vehicle.

In order to achieve this these results will be analysed further and used to inform a second study. This study will be conducted in the coming months at a virtual driving simulator known as DriveLab at the University of Luxembourg. This study will present a driving scenario to users where they will undergo 3 different auditory experiences: 1 . First, drivers will be presented with familiar sounds captured during the field study. 2 . They will then be presented with some of the sounds in 1 but others will be missing. 3. Finally, sounds will be presented that have had 
particular sonic attributes exploited. The order of these experiences will not change, as this will enable the loss of control to be more accurately determined when familiar interior and exterior sounds are removed. Furthermore, this order will enable the altered sonic attributes to be evaluated in terms of how greatly they affect user attention.

\section{References}

[1] Rae, J. B. (1965). The American Automobile: A Brief History. University of Chicago Press.

[2] Ford Model, T. Ford Model T. In Cars (p. 155). PediaPress

[3] Bellis, M. (2010). Automobile history. The history of cars and engines.

[4] Brown, B., \& Laurier, E. (2012). The normal natural troubles of driving with GPS. In ACM Human Factors in Computing Systems (pp. 1621-1630).

[5] Christiansen, L., Frederiksen, N., Jensen, B., Ranch, A., Skov, M., \& Thiruravichandran, N. (2011). Don't look at me, i'm talking to you: investigating input and output modalities for in-vehicle systems. INTERACT 2011, 675-691.

[6] Beuhler, M., Iagnemma, K. \& Singh, S. (2009). The DARPA Urban Challenge: Autonomous Vehicles in City Traffic. London: Springer.

[7] Kim, M. H., Lee, Y. T., \& Son, J. (2010). Age-related physical and emotional characteristics to safety warning sounds: design guidelines for intelligent vehicles. IEEE Transactions, 592-598.

[8] Moore, M., \& Lu, B. (2011). Autonomous Vehicles for Personal Transport: A Technology Assessment.

[9] Lee, J. D., Hoffman, J. D., \& Hayes, E. (2004). Collision warning design to mitigate driver distraction. In Proceedings of the SIGCHI conference on Human factors in computing systems (65-72). ACM.

[10] Tijerina, L., Johnston, S., Parmer, E., Winterbottom, M. D., \& Goodman, M. (2000). Driver distraction with wireless telecommunications and route guidance systems. Washington, DC: U.S. Department of Transportation.

[11] Bishop, R. (2005). Intelligent vehicle technology and trends.

[12] Walker, G. H., Stanton, N. a., \& Young, M. S. (2001). Where Is Computing Driving Cars? ACM CHI 13(2), 203-229.

[13] Stanton, N. A., \& Marsden, P. (1996). From fly-by-wire to driveby-wire: safety implications of automation in vehicles. Safety Science, 24(1), 35-49.

[14] Stanton, N. A., \& Marsden, P. (1996). From fly-by-wire to driveby-wire: safety implications of automation in vehicles. Safety Science, 24(1), 35-49.

[15] Beuhler, M., Iagnemma, K. \& Singh, S. (2009). The DARPA Urban Challenge: Autonomous Vehicles in City Traffic. London: Springer.

[16] Thrun, S. "Google's driverless car." Ted Talk, Ed (2011).

[17] Walker, G. H., Stanton, N. A., \& Young, M. S. (2006). The ironies of vehicle feedback in car design. Ergonomics, 49(2), 161-179.

[18] Kraus, S., Althoff, M., Heissing, B., \& Buss, M. (2009) "Cognition and Emotion in Autonomous Cars," Intelligent Vehicles Symposium, IEEE, Vol., No., pp.635-640, 3-5 June 2009 